Revista Brasileira de

Engenharia Agrícola e Ambiental

v. 15, n.1, p.3-8, 2011

Campina Grande, PB, UAEA/UFCG - http://www.agriambi.com.br

agriambi Protocolo 006.09 - 20/01/2009 • Aprovado em 16/10/2010

\title{
Impacto de los sistemas de riego por goteo en arándanos
}

\author{
Alejandro Pannunzio ${ }^{1}$, Fernando Vilella ${ }^{2}$, Pamela Texeira ${ }^{1} \&$ Zdenka Premuzik ${ }^{3}$
}

\section{RESU MEN}

El ensayo examinó los efectos de diferentes sistemas de riego por goteo en el rendimiento de la variedad 0 ' $\mathrm{N}$ eal de arándano (Vaccinium corymbosum L). El estudio se realizó en Zarate (33 41' S y 59 41' W ), provincia de Buenos Aires, Argentina. El cultivo se implantó en agosto de 2001, la primera cosecha se efectuó en octubre de 2003. El objetivo fue determinar cual era el sistema de riego por goteo que maximizaba el rendimiento, siendo los tratamientos, un lateral de tubería de goteo por fila de plantas con goteros a $40 \mathrm{~cm}$ (T1) y dos laterales de tubería de goteo con goteros a $20 \mathrm{~cm}$ por fila de plantas (T2). El porcentaje de suelo mojado por sistema se incrementa a medida que aumenta el número de emisores por metro lineal. El diseño experimental fue de bloques completamente aleatorizados, con bloques de cinco plantas y cinco repeticiones por tratamiento. La cosecha de 2003, reportó rendimientos de 2436 $\mathrm{kg} \mathrm{ha}^{-1}$ para el tratamiento T1 y de $4335 \mathrm{~kg} \mathrm{ha}^{-1}$ para tratamiento T2. El mojado parcial del suelo que realiza el tratamiento $\mathrm{T1}$, de menor cantidad de goteros por metro lineal, no es suficiente para mojar un porcentaje de suelo compatible con altos rendimientos.

Palabras-clave: rendimiento, volumen de suelo mojado, Vaccinium corymbosum L

\section{Impact of drip irrigation systems in blueberries}

\section{ABSTRACT}

The experiment examined the effects of different drip irrigation systems in an $\mathrm{O}^{\prime} \mathrm{N}$ eal variety of blueberry (Vaccinium corymbosum L). It was contucted at Zarate (33 41' S and 59 41' W ), Buenos Aires, Argentina. Plants of the experiment were planted in August of 2001, after one year in nursery. The first harvest was realized in 0 ctober of 2003. The main scope of the experiment was to find the irrigation system which maximized yields. Treatments were: one drip irrigation line with drippers at $40 \mathrm{~cm}(T 1)$ and two laterals with drippers at $20 \mathrm{~cm}$ per row (T2). The experiment was conducted in a completely randomized block design, each one with 5 plants and 5 replications. During the harvest of $\mathrm{O}$ ctober and $\mathrm{N}$ ovember of 2003, reported yields were $2436 \mathrm{~kg} \mathrm{ha}^{-1}$ for the treatment T1 and $4335 \mathrm{~kg} \mathrm{ha}^{-1}$ for the treatment T2. Treatments that partially irrigate beds are insufficient to obtain high yields.

Key words: yield, soil wetted volume, Vaccinium corymbosum L.

\footnotetext{
${ }^{1}$ Facultad de Agronomía, U niversidad de Buenos Aires. Cátedra de Riego y Drenaje. Av. San Martín 4453 (C1417D SE) Buenos Aires Argentina Fone: +54-11-4524-8000. E-mail: pannunzi@agro.uba.ar; texeira@agro.uba.ar

Cátedra de Producción Vegetal. vilella@agro.uba.ar

3 Cátedra de Química Orgánica. prezumik@agro.uba.ar
} 


\section{INTRODUCCIÓN}

El cultivo de arándano se introdujo en América del Sur, en la década de los años '80, para evaluar su potencial en la región. Desde entonces la industria del arándano creció muy rápidamente en Chile y Argentina (Bañados, 2006).

El área del ensayo recibe alrededor de $1000 \mathrm{~mm}$ de precipitaciones anuales, con una distribución irregular dentro del año, que genera déficit hídrico para las plantas, sobre todo si se tiene en cuenta el escaso desarrollo radicular del arándano (Vaccinium corymbosum L).

Existen reportes del incremento de rendimientos para diferentes frutales en zonas húmedas, con la incorporación de sistemas de riego localizado. Un aspecto importante al considerar el riego localizado es la selección de los emisores correctos en base a distintos factores (Holpzafel et al., 2007).

El máximo potencial de producción del arándano está asociado a varios factores (Pannunzio, 2008), entre los más relevantes se consideran el marco de plantación (Strik \& Buller, 2005), la variedad (Bryla \& Strik, 2007), el manejo del riego (Holzapfel et al., 2004; Bryla, 2006), la fertilización (Pannunzio et al., 2009; Pannunzio \& Texeira, 2009; Bryla \& Machado, 2007), y el pH (Ferreyra et al., 2001).

Los suelos de la zona son de textura franco arcillosa limosa, con un gran aporte de materia orgánica constituido básicamente por corteza de pino, chips de diversos orígenes, cama de pollo, etc., con la finalidad de generar un medio ácido, muy aireado y con alto contenido de materia orgánica, similar al lugar de origen del arándano. El área donde se desarrolló el cultivo es un camellón de $40 \mathrm{~cm}$ de altura y 60 a $100 \mathrm{~cm}$ de ancho, con estructura trapezoidal.

La distribución del agua dentro del suelo tiene un efecto importante en la producción de arándanos, de modo que el riego es un factor a considerar dentro del manejo del cultivo, principalmente por el sistema radicular superficial de esta especie. Estudios recientes, muestran incrementos de hasta el $43 \%$ en el rendimiento de arándano, con la aplicación de riego (Yarborough, 2004; Seymour et al., 2004).

El manejo del potencial agua del suelo es importante para lograr altas tasas de crecimiento, siendo $10 \mathrm{cb}$, el potencial aconsejado para el mismo. La mayor demanda de agua ocurre previo a la cosecha con evapotranspiraciones reales de $19 \mathrm{~L}$ planta $^{-1}$ para cultivos regados por goteo (Jaureguiberry, 1991). La determinación del coeficiente de cultivo (kc) es fundamental en el manejo del riego (Starr et al., 2004). El coeficiente de cultivo (kc) para cultivos de un año es de 0,2; también se cita un kc 0,97 para riego por goteo en arándanos de tres años (Riveros, 1996). Los autores Bryla \& Strik (2007), proponen una evolución del coeficiente de cultivo ( $\mathrm{kc}$ ) de 0,2 a 1,1. Holzapfel et al. (2004) encontraron requerimientos de alrededor de $750 \mathrm{~mm}$ anuales en la zona de Chillán-Chile. Un importante factor que es necesario considerar debido a que influye sobre los requerimientos hídricos, es la densidad de plantación (Bryla \& Strik, 2005).

La selección y la gestión de sistemas de riego es de suma importancia en el arándano (Holzapfel, 2009; Holzapfel et al., 2007). Tanto un déficit como un exceso de agua tienen un efecto depresivo sobre el crecimiento de las plantas. El déficit de agua, no permite que la planta alcance su rendimiento potencial, la sensibilidad a los déficits hídricos dependerá de su estado fenológico (Mingeau et al., 2001). Por otro lado, el exceso de agua, puede favorecer el desarrollo de ciertas enfermedades (Bryla \& Linderman, 2007).

\section{Materiales y MÉTODOS}

\section{Ubicación}

El experimento fue realizado en la ruta provincial 193, km 14,5, zona de Zarate, provincia de Buenos Aires, República Argentina, a una latitud de $33^{\circ} 41^{\prime}$ 'S y $59^{\circ} 41^{\prime}$ W de longitud, la altura sobre el nivel del mar es de $28 \mathrm{~m}$. El estudio se llevó a cabo en un establecimiento de 15 hectáreas, dedicado exclusivamente a la producción de arándanos desde 1997.

\section{Clima}

La zona se caracteriza por una temperatura media de $20,1^{\circ} \mathrm{C}$, una máxima de $27,5^{\circ} \mathrm{C}$ y una mínima de $13,8^{\circ} \mathrm{C}$, respectivamente. La máxima absoluta registrada es de $40{ }^{\circ} \mathrm{C}$ y la mínima de $-6^{\circ} \mathrm{C}$. Con respecto al periodo libre de heladas, ocurren de 6 a 9 heladas anuales. Las precipitaciones medias anuales son de $1050 \mathrm{~mm}$.

\section{Suelo}

Son argiudoles, con un horizonte $\mathrm{A}$, de $20 \mathrm{~cm}$ de profundidad, un $\mathrm{B}_{2 \mathrm{t}}$ de 20 a $40 \mathrm{~cm}$, un $\mathrm{C}_{1}$ de 40 a $70 \mathrm{~cm}$. La infiltración básica de estos suelos es de $13 \mathrm{~mm} \mathrm{~h}^{-1}$. En la Tabla 1 pueden observarse los datos de composición textural, los valores de $\mathrm{pH}$ y salinidad de los mismos.

Tabla 1. Composición textural, pH y CE del suelo del estudio

\begin{tabular}{|c|c|c|c|}
\hline \multirow{2}{*}{ Características } & \multicolumn{3}{|c|}{ Profundidad em cm } \\
\hline & $0-20$ & $20-40$ & $40-60$ \\
\hline Horizonte & $A$ & B & C \\
\hline Área \% & 14 & 13 & 10,5 \\
\hline Limo \% & 66 & 57 & 52,0 \\
\hline Arcilla \% & 20 & 30 & 37,5 \\
\hline Textura & franco limosa & $\begin{array}{c}\text { franco arcillo } \\
\text { limosa }\end{array}$ & $\begin{array}{c}\text { franco arcillo } \\
\text { limosa }\end{array}$ \\
\hline $\mathrm{pH}$ & 5,7 & 5,9 & 6,2 \\
\hline CE mm hos $\mathrm{cm}^{-1}$ & 0,3 & 0,3 & 0,2 \\
\hline
\end{tabular}

\section{Agua de riego}

El agua de riego es de origen subsuperficial, proviene del acuífero de Arenas Puelches. Su extensión es del orden de $240.000 \mathrm{~km}^{2}$ en las provincias de Santa Fe, Entre Ríos, Córdoba y Buenos Aires; en esta última ocupa unos $92000 \mathrm{~km}^{2}$. Los espesores del acuífero son de 10 a $40 \mathrm{~m}$ de profundidad. Las aguas son bicarbonatadas sódicas (Amato \& Busso, 2006), con valores de salinidad de 700 a 1100 micromhos $\mathrm{cm}^{-1}$, y valores de $\mathrm{pH}$ de 7 a 7,7. El espesor del Puelche es de $20 \mathrm{~m}$ en el área de estudio, llega hasta $90 \mathrm{~m}$ en otras áreas de su extensión, aumentando ligeramente hacia los ríos Paraná - de la Plata y marcadamente hacia la cuenca del Salado y el Cabo San Antonio. Se encuentra delimitado superiormente por un 
acuitardo con una transmisividad del orden de $5.10^{-4} \mathrm{~d}^{-1}$ e inferiormente, por un acuícludo que lo separa del Acuífero Paraná.

\section{Plantación}

La variedad de arándanos utilizada para el ensayo fue O`Neal, que representaba el $80 \%$ de la superficie plantada a la fecha del estudio en Argentina. La plantación se realizó con camellones distanciados 3 metros entre sí y con plantas distanciadas a $75 \mathrm{~cm}$, determinando una densidad de 4444 plantas por hectárea.

\section{Sistemas de riego}

Los sistemas de riego evaluados, fueron presurizados del tipo goteo. El primer sistema de goteo es una tubería de $16 \mathrm{~mm}$ de diámetro, con goteros a $40 \mathrm{~cm}$ de $1,7 \mathrm{~L} \mathrm{~h}^{-1}$ de caudal a $10 \mathrm{~m}$ de columna de agua de presión, marca Naan de Israel, modelo Naan Paz. El segundo tratamiento incluye dos laterales de goteo por fila de plantas, cada uno de ellos con emisores a $20 \mathrm{~cm}$ de distancia entre si de $1,0 \mathrm{~L} \mathrm{~h}^{-1}$ de caudal, marca Aquatraxx, fabricada por Toro de USA.

El potencial agua utilizado para el ensayo fue de $10 \mathrm{cb}$. La lámina de riego utilizada fue de $5 \mathrm{~mm} \mathrm{~d}^{-1} \mathrm{y}$ fue la misma para los dos tratamientos, la variable de ajuste para lograr esto fue el tiempo de riego.

\section{Diseño experimental}

El diseño experimental fue en bloques completamente aleatorizados (DBCA). Se evaluaron dos tratamientos de riego, el ensayo incluyó cinco repeticiones por tratamiento, con bloques de cinco plantas cada uno. Los tratamientos evaluados fueron, $\mathrm{T} 1$ con un lateral de riego por fila de plantas con goteros de $1,7 \mathrm{~L} \mathrm{~h}^{-1}$ a $40 \mathrm{~cm}$, y T2 con dos laterales por fila de plantas con goteros de $1,0 \mathrm{~L} \mathrm{~h}^{-1}$ a cada $20 \mathrm{~cm}$. El caudal por metro lineal fue de 4,25 y 10,0 L h ${ }^{-1}$, respectivamente. Estos caudales, determinaron diferentes áreas de suelo mojadas por tratamiento. La dosis de fertilizantes fue la misma para ambos tratamientos y su aplicación se realizó independientemente del sistema de riego. Los parámetros determinados para cada tratamiento fueron: producción por día, producción acumulada, precios de mercado de Miami (USA) de la fruta cosechada, ingreso neto según fecha, precio neto e ingreso neto para el productor por tratamiento.

\section{RESULTADOS Y DISCUSIÓN}

En la Figura 1, puede observarse la producción acumulada en $\mathrm{kg} \mathrm{ha}^{-1}$, según la fecha de cosecha, para los tratamientos T1 y $\mathrm{T} 2$, desde las primeras fechas se observa una diferencia entre ambos tratamientos; esta diferencia se incrementa a medida que se avanza en la cosecha. En los finales de la cosecha se registran valores de $2436 \mathrm{~kg} \mathrm{ha}^{-1}$ para el tratamiento $\mathrm{T} 1 \mathrm{y}$ de $4335 \mathrm{~kg} \mathrm{ha}^{-1}$ para el tratamiento T2.

Los resultados son coincidentes con los encontrados en otros cultivos, los que indican que a mayor superficie de mojado corresponden mayores rendimientos (Morales et al., 2010; García Petillo \& Castel, 2004; Gispert, 2003; Andiloro, 1997;

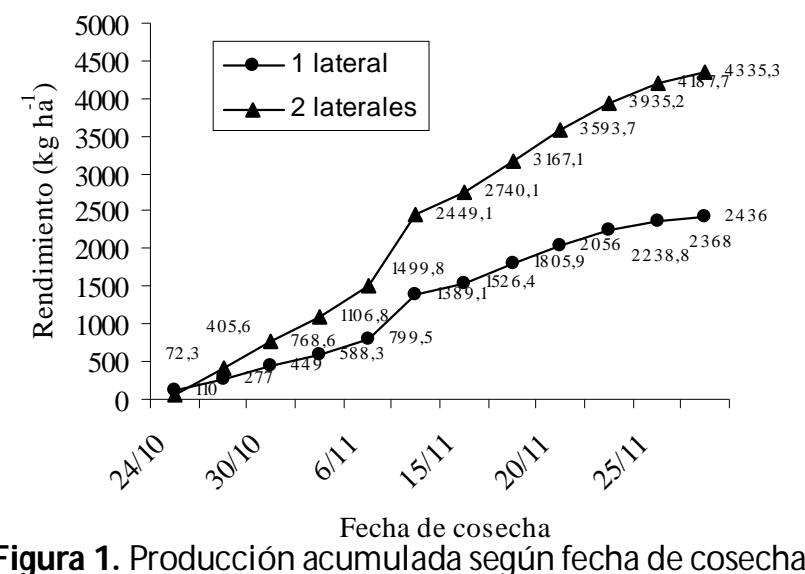

Castel \& Buj, 1993). En cultivos de arándanos implantados sobre camellones de 60 a $100 \mathrm{~cm}$ de ancho, la mayor superficie de mojado se lograría utilizando un sistema de doble lateral de tubería de goteo, con goteros lo suficientemente cercanos entre si, como para distribuir el agua en toda la superficie donde se desarrollan las raíces.

En el caso de un único lateral de riego una parte importante del camellón no fue mojado. En cultivos que desarrollan sus raíces muy superficialmente, como es el caso del cultivo de arándano, que tiene raíces de no más de $20 \mathrm{~cm}$, una reducción del área mojada puede conducir a un severo déficit hídrico, con graves reducciones sobre la magnitud de la evapotranspiración real (Hunt et al., 2008; Allen et al., 2000).

En cambio, el T2, con dos laterales, mojó casi todo el camellón. Esto generó al comienzo, pérdidas de agua, ya que las raíces no se encontraban en toda la superficie mojada y una porción del agua lixivió en profundidad, durante el primer verano. Cuando las plantas de arándano llegan al campo desde el vivero, están contenidas en macetas que oscilan, según el caso, entre 2 y 3 L de capacidad, el volumen de raíces es, en ambos casos, reducido, comparado con $\operatorname{los} 75 \mathrm{~cm}$, entre plantas y los 60 a $100 \mathrm{~cm}$ de ancho que tendrán para desarrollarse al ser adultas. Se observó que el potencial agua en el suelo alcanzó valores no compatibles con la necesidad de los cultivos, si solo se consideraban los aportes de agua por precipitaciones.

Como se mencionó anteriormente, el tratamiento T2, al mojar todo el camellón, favoreció un sistema radicular más extenso. En cambio, el tratamiento T1 que sólo mojó una porción del camellón, no llegó a cubrir toda el área que desarrollaron las raíces, como consecuencia de las precipitaciones, originándose una situación de déficit hídrico para estas plantas.

En la Figura 2, se observa el ingreso neto para los distintos tratamientos, la tendencia de las curvas es la misma que la registrada para el rendimiento. Hacia finales de la fecha de comercialización, se registraron valores de U\$S 48843 para el tratamiento T2 y de U\$S 27449 para el tratamiento T1. Si bien en el presente estudio, no se comparó la inversión inicial para ambos sistemas, es importante aclarar que el tratamiento T2 requerirá de una inversión mayor.

En la Tabla 2, se incluyen las fechas de cosecha del ensayo y los precios del mercado de Miami, correspondientes a tres días posteriores y coincidentes con la fecha de comercialización, considerando el tiempo desde cosecha hasta llegar a Miami. 


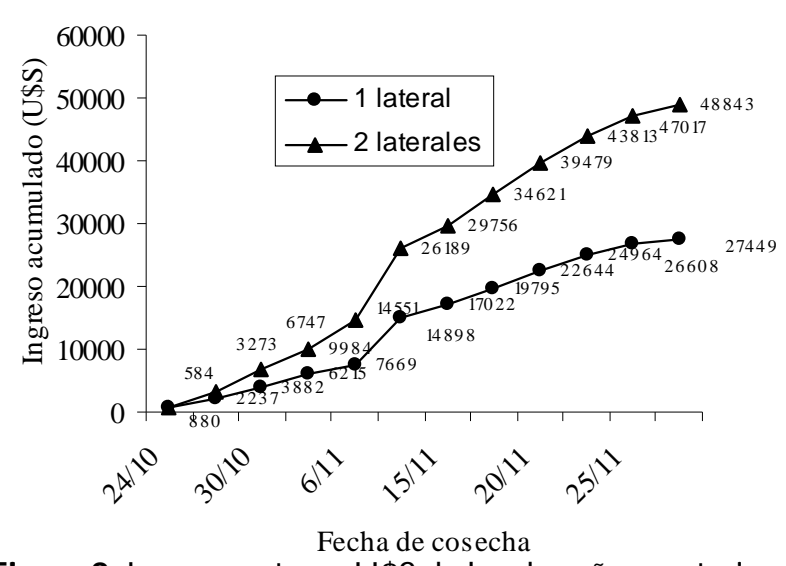

Figura 2. Ingreso neto en U $\$ S$ de los dos años posteriores al transplante

Tabla 2. Fechas de cosecha, comercialización y precios de venta

\begin{tabular}{ccc}
\hline $\begin{array}{c}\text { Fecha de } \\
\text { cosecha }\end{array}$ & $\begin{array}{c}\text { Fecha de } \\
\text { comercialización }\end{array}$ & $\begin{array}{c}\text { Precio promedio }(\text { US kg } \\
\text { para cajas de 1,5 } \\
\text { en Miami, USA }\end{array}$ \\
$24 / 10 / 2003$ & $27 / 10 / 2003$ & 15,2 \\
$26 / 10 / 2003$ & $27 / 10 / 2003$ & 15,2 \\
$30 / 10 / 2003$ & $31 / 10 / 2003$ & 17,5 \\
$03 / 11 / 2003$ & $03 / 11 / 2003$ & 17,5 \\
$06 / 11 / 2003$ & $07 / 11 / 2003$ & 20,7 \\
$10 / 11 / 2003$ & $12 / 11 / 2003$ & 21,7 \\
$15 / 11 / 2003$ & $17 / 11 / 2003$ & 21,7 \\
$17 / 11 / 2003$ & $19 / 11 / 2003$ & 22,0 \\
$20 / 11 / 2003$ & $21 / 11 / 2003$ & 22,0 \\
$22 / 11 / 2003$ & $24 / 11 / 2003$ & 22,3 \\
$25 / 11 / 2003$ & $26 / 11 / 2003$ & 22,3 \\
$29 / 11 / 2003$ & $01 / 12 / 2003$ & 21,8 \\
\hline
\end{tabular}

En la Tabla 3, se realizó el cálculo del ingreso neto al productor deduciendo los costos de cada etapa de comercialización desde la cosecha hasta la venta en USA. La Tabla 4 muestra el resumen del ingreso neto por kg para cada fecha de cosecha.

El sistema de doble lateral resulta particularmente conveniente para plantaciones con camellones de estructura trapezoidal, donde se pueden ubicar los dos laterales de riego,
Tabla 4. Ingreso neto por kg, según fechas de cosecha y comercialización

\begin{tabular}{ccc}
\hline Fecha cosecha & Precio promedio $\left(\mathbf{U} \mathbf{S} \mathbf{~ k g}^{-1}\right)$ & Ing. Neto $\left(\mathbf{U} \mathbf{S} \mathbf{~ k g}^{-1}\right.$ ) \\
$24 / 10 / 2003$ & 15,17 & 8,07 \\
$26 / 10 / 2003$ & 15,17 & 8,07 \\
$30 / 10 / 2003$ & 17,50 & 9,57 \\
$03 / 11 / 2003$ & 17,50 & 9,57 \\
$06 / 11 / 2003$ & 20,67 & 11,62 \\
$10 / 11 / 2003$ & 21,67 & 12,26 \\
$15 / 11 / 2003$ & 21,67 & 12,26 \\
$17 / 11 / 2003$ & 22,00 & 11,39 \\
$20 / 11 / 2003$ & 22,00 & 11,39 \\
$22 / 11 / 2003$ & 22,33 & 12,69 \\
$25 / 11 / 2003$ & 22,33 & 12,69 \\
$29 / 11 / 2003$ & 21,83 & 12,37 \\
\hline Fuente: USDA y North Bay Argentina S.A.
\end{tabular}

lo suficientemente distanciados como para mojar una gran superficie, sin que el agua escurra del camellón al entresurco, situación que ocurriría en el caso de los camellones triangulares.

Con respecto a la fecha de comienzo y finalización de cosecha no se observaron diferencias entre los distintos tratamientos, no pudiendo sacarse conclusiones sobre este punto.

Es importante considerar que otros autores encontraron que la aspersión y la microaspersión, permiten obtener frutas más firmes y con mayor contenido de sólidos solubles que el goteo (Bryla et al., 2009). Por lo tanto, bajo ciertas circunstancias, la aspersión y microaspersión podrían mejorar el almacenamiento y la calidad de la fruta para el mercado.

Por otro lado, el riego por goteo permite una mayor eficiencia en el uso del agua, en comparación con otros sistemas, las plantas regadas por microaspersión requieren un $130 \%$ más de agua que las regadas por goteo. (Bryla et al., 2009). Los resultados hallados, contribuyen a mejorar el manejo del riego por goteo, con el objetivo de mantener un alto nivel de productividad. Estos puntos considerados, son requisitos básicos para el logro de un agroecosistema económico y ambientalmente sustentable.

Tabla 3. Cálculo del Ingreso $\mathrm{N}$ eto al productor

\begin{tabular}{|c|c|c|c|c|c|c|c|}
\hline Fecha de comercialización & $27 / 10 / 2003$ & $\begin{array}{l}31 / 10 / 2003 \\
03 / 11 / 2003\end{array}$ & 07/11/2003 & $\begin{array}{l}12 / 11 / 2003 \\
17 / 11 / 2003\end{array}$ & $\begin{array}{l}19 / 11 / 2003 \\
21 / 11 / 2003\end{array}$ & $\begin{array}{l}24 / 11 / 2003 \\
26 / 11 / 2003\end{array}$ & $01 / 12 / 2003$ \\
\hline Precio venta Miami por caja $1,5 \mathrm{~kg}$ & 22,76 & 26,25 & 31,01 & 32,51 & 33,00 & 33,35 & 32,75 \\
\hline Gastos en Miami & $-0,80$ & $-0,80$ & $-0,80$ & $-0,80$ & $-0,80$ & $-0,80$ & $-0,80$ \\
\hline Comissión del importador $(8,5 \%)$ & $-1,93$ & $-2,23$ & $-2,64$ & $-2,76$ & $-2,81$ & $-2,83$ & $-2,78$ \\
\hline Precio CIF Miami, por caja $1,5 \mathrm{~kg}$ & 20,02 & 23,22 & 27,57 & 28,94 & 29,40 & 29,71 & 29,16 \\
\hline Fletes y seguro aéreo, por caja 1,5 kg & $-3,00$ & $-3,00$ & $-3,00$ & $-3,00$ & $-3,00$ & $-3,00$ & $-3,00$ \\
\hline Precio FOB por caja 1,5 kg & 17,02 & 20,22 & 24,57 & 25,94 & 26,40 & 26,71 & 26,16 \\
\hline Flete interno & $-0,30$ & $-0,30$ & $-0,30$ & $-0,30$ & $-0,30$ & $-0,30$ & $-0,30$ \\
\hline Fumigación y empaque & $-0,80$ & $-0,80$ & $-0,80$ & $-0,80$ & $-0,80$ & $-0,80$ & $-0,80$ \\
\hline Materiales empaque & $-0,97$ & $-0,97$ & $-0,97$ & $-0,97$ & $-0,97$ & $-0,97$ & $-0,97$ \\
\hline Gastos despacho y Aduana & $-0,18$ & $-0,18$ & $-0,18$ & $-0,18$ & $-0,18$ & $-0,18$ & $-0,18$ \\
\hline Comissión exportadora (7,65\% s/FOB) & $-1,30$ & $-1,55$ & $-1,88$ & $-1,98$ & $-2,02$ & $-2,04$ & $-2,00$ \\
\hline Gastos empaque y consecha & $-0,80$ & $-0,80$ & $-0,80$ & $-0,80$ & $-0,80$ & $-0,80$ & $-0,80$ \\
\hline Retorno al productor por caja 1,5 kg & 12,67 & 15,62 & 19,64 & 20,91 & 21,33 & 21,62 & 21,11 \\
\hline Retorno al productor por kg & 8,45 & 10,41 & 13,09 & 13,94 & 14,22 & 14,41 & 14,07 \\
\hline \% retorno al productor según precio venta & 55,67 & 59,51 & 63,34 & 64,32 & 64,62 & 64,83 & 64,47 \\
\hline
\end{tabular}

Fuente: USDA y North Bay Argentina S.A. 


\section{Conclusiones}

1. Los resultados obtenidos sugieren la conveniencia de regar mediante los sistemas de riego localizado, una superficie acorde al área donde se desarrollan las raíces del cultivo.

2. Si bien el sistema de doble lateral generó algunas pérdidas de agua cuando el cultivo era muy pequeño y sus raíces no ocupaban todo el camellón, el resultado medido a través de su producción, en la primera cosecha arrojó resultados favorables.

\section{ACLARACIÓN}

El hecho de mencionar marcas comerciales obedeció solamente a la necesidad de identificar los materiales empleados en el ensayo, no existiendo ninguna recomendación ni relación entre los autores y dichas firmas comerciales.

\section{AgradeCimientos}

Al señor Darío Calderón, sin cuya inestimable y desinteresada colaboración, este trabajo de investigación, no podría haberse llevado adelante.

\section{LITERATURA CITADA}

Allen, L.; Brakke M.; Baker J.; Jones J. Gas exchange and biomass responses of young citrus trees to partial rootingvolume irrigation. Proceedings of Soil and Crop Science Society of Florida, v.59, p.37-45, 2000.

Amato, S.; Busso, S. Relaciones estratigráficas e hidroquímicas de los acuíferos Pampeano y Puelches en el noreste de la provincia de Buenos Aires. Revista del Museo Argentino de Ciencias Naturales, v.8, p.9-26, 2006.

Andiloro, S. Effect of various irrigation methods on the growth and productivity of oranges in the Gioia Tauro Plain, Italy. Irrigazione e Drenaggio, v.44, p.26-32, 1997.

Bañados, M. Blueberry production in South America. Acta Horticulturae, v.715, p.165-172, 2006.

Bryla, D. R. Drip irrigation configuration influences growth in young highbush blueberries. Hort Science, v.41, p.1012, 2006.

Bryla, D. R.; Linderman, R. Implications of irrigation method and amount of water application on Phytophtora and Pytium infection and severity of root rot in highbush blueberry. Hort Science, v.42, p.1463-1467, 2007.

Bryla, D. R; Machado, R. Nitrogen fertigation is less efficient but safer than granular fertilizer application in newly-planted blueberry. Hort Science, v.42, p.1021, 2007.

Bryla, D. R., Strik, B. E. Water requirements of highbush blueberry planted at normal and high density spacings. Hort Science, v.40, p.1058-1059, 2005.

Bryla, D. R.; Strik B. E. Effects of cultivar and plant spacing on the seasonal water requirements of highbush blueberry. Hort Science, v.132, p.270-277, 2007.
Bryla, D. R.; Yorgey, B.; Shireman, A. Irrigation management effects on yield and fruit quality of highbush blueberry. Acta Horticulturae, v.810, p.649-656, 2009.

Castel, J; Buj, A. Riego por goteo deficitario en naranjos adultos "Salustiana" durante siete años. Revista Investigación Agraria: Producción y Protección Vegetal, v.8, p.191-204, 1993.

Ferreyra, R.; Peralta, J.; Sadzawka, A.; Muñoz, C.; Valenzuela, J. Efecto de la acidificación del sustrato y del agua de riego en la nutrición, desarrollo y producción de arándano ojo de conejo (Vaccinium ashei reade). Agricultura Técnica, v.61, p.452-458, 2001.

García Petillo, M.; Castel, J. The response of Valencia orange trees to irrigation in Uruguay. Spanish Journal of Agricultural Research, v.2, p.429-443, 2004.

Gispert, J. Evaluación del volumen de suelo húmedo en microirrigación. Influencia del porcentaje de este volumen sobre el comportamiento del olivo (Olea Europea L. cult. "Arbequina"). Estudios de la Zona no Saturada del Suelo, v.6, p.51-57, 2003.

Holzapfel, E. Selection and management of irrigation systems for blueberry. Acta Horticulturae, v.810, p.641-648, 2009.

Holzapfel, E.; Abarca W.; Paz, V.; Arumi, J.; Rodriguez, A.; Orrego, X.; Lopez, M. Selección técnico-económica de emisores. Revista Brasileira de Engenharia Agricola e Ambiental, v.11, p.547-556, 2007.

Holzapfel, E.; Hepp R.; Mariño M. Effect of irrigation on fruit production in blueberry. Agricultural Water Management, v.67, p 173-184, 2004.

Hunt, J.; Honeycutt W.; Starr G.; Yarborough, D. Evapotranspiration rates and crop coefficients for lowbush blueberry (Vaccinium angustifolium). International Journal of Fruit Science, v.88, p.282-298, 2008.

Jaureguiberry, P. Relaciones agua crecimiento en arándano alto (Vaccinium corimbosum L.) regado por microaspersión y goteo. Chillan: Facultad de Agronomía, Universidad de Concepción, 1991. 143p. Tesis Grado

Mingeau, M.; Perrier, C.; Améglio, T. Evidence of droughtsensitive periods from flowering to maturity on highbush blueberry. Scientia Horticulturae, v.89, n.1, p.23-40, 2001.

Morales, P.; García Petillo, M; Hayashi, R.; Puppo, L. Respuesta del duraznero a diferentes patrones de aplicación del agua. Revista Brasileira de Engenharia Agrícola e Ambiental, v.141, n.1, p.17 - 24, 2010.

Pannunzio, A. Efectos de sustentabilidad de los sistemas de riego en arándano. Buenos Aires: Facultad de Ciencias Veterinarias/UBA, 2008. 125p. Tesis Maestría

Pannunzio, A.; Texeira, P. Respuesta del arándano al riego por goteo y a la aplicación de calcio y potasio. En: Simposio Internacional de Arándanos y Berries y Congreso Latinoamericano de Arándanos y Berries, 5, 2008, Buenos Aires. Anales... Buenos Aires: Facultad de Agronomía de la Universidad de Buenos Aires, 2008. p.123-133. 
Pannunzio, A.; Texeira, P.; Vilella, F.; Puhl, L. Fertigation in blueberries in Concordia, Argentina. Acta Horticulturae, v.810, p.771-776, 2009.

Riveros, C. Respuesta del arándano alto (Vaccinium corymbosum L.) de tercer año [cv Bluetta] al nivel de agua aplicado bajo riego por goteo y microjet. Chillan: Facultad de Agronomía/Universidad de Concepción, 1996. 3p. Tesis Grado

Seymour, M.; Starr, G.; Yarborough, D. Lowbush Blueberry (Vaccinium angustifolium) with irrigated and rain-fed conditions. Small Fruits Review, v.33, p.45-56, 2004.
Starr, G.; Seymour, R.; Olday, F.; Yarborough, D. Determination of evapotranspiration and drainage in lowbush blueberries (Vaccinium angustifolium) using weighing lysimeters. Small Fruits Review, v.33, p.273283, 2004.

Strik, B.; G. Buller. The impact of early cropping on subsequent growth and yield of highbush blueberry in the establishment years at two planting densities is cultivar dependent. Hort Science, v. 40, p.1998-2001, 2005.

Yarborough, D. Factors contributing to the increase in productivity in the wild blueberry industry. Small Fruits Review, v.33, p.33-43, 2004. 